\title{
Pallister-Hall syndrome
}

INSERM

\section{Source}

INSERM. (1999). Orphanet: an online rare disease and orphan drug data base. PallisterHallsyndrome. ORPHA:672

Pallister-Hall syndrome (PHS), a pleiotropic autosomal dominant malformative disorder, is characterized by hypothalamic hamartoma, pituitary dysfunction, bifid epiglottis, polydactyly, and, more rarely, renal abnormalities and genitourinary malformations. 